PAWE⿺ MATYASZEWSKI

ORCID: 0000-0001-6214-6871

Université catholique de Lublin Jean-Paul II

p.matyaszewski@chello.pl

\title{
LE VOYAGE UTOPIQUE DE WOJCIECH GUTKOWSKI : LA POLOGNE SE TROUVE-T-ELLE EN AUSTRALIE?
}

Il faut avouer que non seulement l'œuvre, mais aussi la personne, jusqu'au nom même de Wojciech Gutkowski (1775-1826), sont aujourd'hui presque complètement oubliés en Pologne. À vrai dire, seuls quelques rares spécialistes en littérature ou en histoire des idées connaissent certains éléments de la biographie et quelques-uns des écrits de cet ancien noble polonais, officier d'artillerie, économiste, franc-maçon, plutôt écrivain publiciste qu'homme de lettres, auteur d'un seul ouvrage romanesque ${ }^{1}$. C'est d'ailleurs grâce à ce dernier que l'on veut encore se souvenir de lui, tellement le nom de Gutkowski doit évoquer inévitablement, du moins pour ceux qui savent l'identifier, le titre de son opus vitae, le roman Podróż do Kalopei [Le Voyage en Kalopée]. Non seulement l'ouvrage n'a jamais été traduit en français mais, qui plus est, il n'a vu le jour en Pologne que très tardivement, en 1956, soit 130 ans après la mort de son auteur ${ }^{2}$. Présenté

${ }^{1}$ Cf. M. Tyrowicz, Gutkowski Wojciech, [dans :] Polski Stownik Biograficzny, t. IX/1, f. 40, Wrocław 1960, pp. 182-183 ; J. Bartyś, Wizjoner czy fantasta. O życiu i działalności Wojciecha Gutkowskiego (1775-1826), Wydawnictwo Lubelskie, Lublin 1983 ; Z. Najdowski, „Podróż do Kalopei” Wojciecha Gutkowskiego. Studium z dziejów myśli polskiego Oświecenia, PWN, Warszawa 1988.

${ }^{2}$ Cf. W. Gutkowski, Podróż do Kalopei, opracował i wstępem opatrzył dr Zygmunt Gross, PWN, Warszawa 1956. Le texte de Gutkowski, précédé d'une longue introduction de Zygmunt Gross (pp. 5-51), se trouve aux pages 55-264. Toutes les citations, traduites en français par l'auteur de la présente étude, viennent de cette édition du roman de Gutkowski, à laquelle nous renvoyons par l'abréviation PdK. 
par Gutkowski lui-même en 1817 devant la Société des Amis des Sciences de Varsovie dans l'espoir aussi ferme que naïf de le voir publié par la suite, le manuscrit du roman a été accueilli par le sage aréopage polonais avec beaucoup de réserves et, malheureusement, déposé dans le fonds impénétrable de la Société, condamné ainsi à de longues années de silence et d'oubli.

Afin de comprendre (et là, il faut déclarer tout de suite que « comprendre » ne veut nullement signifier « approuver »), les raisons pour lesquelles la Société des Amis des Sciences de Varsovie a refusé, pour ne pas dire désavoué vivement le roman de Wojciech Gutkowski il y a deux siècles, il faut avant toute chose le présenter brièvement. Il s'agit d'un récit de voyage typique de l'époque des Lumières, surtout de la charnière des $\mathrm{XVIII}^{\mathrm{e}}$ et $\mathrm{XIX}^{\mathrm{e}}$ siècles, raconté à la première personne par le narrateur lui-même (ce qui doit renforcer l'impression d'authenticité de la fiction $)^{3}$, ici un jeune noble polonais anonyme, dont on ne sait même pas de quelle partie de la Pologne il vient. Celui-ci, sans doute alter ego de Wojciech Gutkowski, décide en 1796, après le troisième partage, c'est-à-dire après la disparition de la Pologne de la carte géopolitique de l'Europe, de quitter son pays natal et de « voyager à travers le monde », afin d'atténuer, en vrai romantique, l'« état de mélancolie »dans lequel il est tombé suite aux malheurs de sa patrie (PdK, p. 66). Après avoir visité quelques pays d'Europe (dont il ne dévoile pourtant pas les noms) et s'être arrêté quelque temps au Cap de Bonne Espérance et dans différentes îles de l'Océan Indien, il arrive enfin aux Indes Orientales. Là, il rencontre des « ingénieurs » français (en fait, comme le narrateur l'expliquera d'ailleurs plus loin, il s'agit davantage de savants : ce sont des cartographes, botanistes, zoologistes, minéralogistes) qui s'apprêtent à partir pour la Nouvelle Hollande pour au moins deux ans. Il n'hésite pas à s'embarquer avec eux et, en 1802, il pose le pied sur le sol australien à hauteur du Golfe Joseph Bonaparte, c'est-à-dire dans la partie Sud de l'Australie. Il faut rappeler ici que cette dernière a porté jusqu'en 1824 le nom de Nouvelle Hollande, comme l'avait appelée en 1644 le voyageur et explorateur hollandais Abel Tasman (1603-1659). L'allusion que fait Gutkowski à des ingénieurs français qui se rendent en Nouvelle Hollande permet de supposer qu'il pense peut-être ici à la fameuse expédition de Nicolas Baudin (1754-1803), cartographe et voyageur français parti en 1800 pour un voyage d'exploration de trois ans sur les côtes de l'Australie, avec vingt-deux savants français (botanistes, zoologistes, minéralogistes, cartographes, dessinateurs). Cette expédition importante

${ }^{3}$ Comme l'explique très bien Jean-Michel Racault, « le recours au récit à la première personne et à l'effet qui s'y attache : le je du voyageur-narrateur, garantit la réalité de ses aventures. De plus, le souci de rendre 'vraisemblable' le récit permet de l'inscrire dans la perspective documentaire de la relation du voyage véridique » (cf. J.-M. Racault, Nulle part et ses environs. Voyage aux confins de l'utopie littéraire classique (1657-1802), PUF, Paris 2003, p. 379). Sur la fiction littéraire à la première personne de l'époque du tournant des Lumières en France, voir surtout : A. Rabsztyn, L'hybridité du roman français à la première personne (1789-1820), Wydawnictwo Uniwersytetu Śląskiego, Katowice 2017. 
partie à bord de deux navires, Le Géographe et Le Naturaliste, a apporté des fruits inestimables pour la connaissance des Terres Australes par les Européens au début du XIX ${ }^{\mathrm{e}}$ siècle ${ }^{4}$. Il faut remarquer que non seulement l'époque de cette expédition correspond exactement à celle où le narrateur polonais rencontre sur son chemin les voyageurs français, mais aussi, que c'est Nicolas Baudin qui, en 1802, a baptisé une partie du littoral australien le Golfe Joseph Bonaparte, nom qui apparaît explicitement dans le texte du romancier polonais (PdK, p. 56).

$\mathrm{Si}$, apparemment, le début de l'ouvrage de Gutkowski n'a pas l'air d'avoir de quoi inquiéter les membres de la Société des Amis des Sciences de Varsovie, il faut avancer plus dans la lecture comme dans le voyage pour comprendre, toujours sans l'approuver, leur décision d'enterrer dans les archives le manuscrit de son roman. Arrivé sur la côte, le narrateur décide peu de temps après d'abandonner ses compagnons de route français afin de pénétrer dans l'intérieur de l'île australienne qui, comme il le dit, est « presque aussi grande que l'Europe tout entière et, jusque là, demeure inexplorée » (PdK, p. 70). Ayant traversé des montagnes et surmonté plusieurs rivières, ruisseaux et cataractes, il se retrouve devant une chaîne immense de forêts impénétrables. Après huit jours de marche extrêmement difficile, entouré de roches dangereuses et d'animaux féroces, il arrive sur les bords d'une rivière où il découvre une colonne maçonnée sur laquelle se trouve gravée une représentation du soleil et une inscription rédigée en quatre langues : en polonais d'abord, puis en latin, en français et en anglais, qui dit la même chose dans toutes ces langues, à savoir «Frontière des Kalops» (PdK, p. 61). Étonné de la présence de ces langues, surtout du polonais, au centre de l'Australie sauvage, et de la connaissance des sciences et des arts dont fait preuve la colonne, exécutée « en toute perfection selon les normes de sculpture et de peinture » (PdK, p. 61), il décide d'avancer et de pénétrer dans l'inconnu mystérieux. Et c'est ici que commence le véritable sens du roman et que se laisse dévoiler son caractère utopique, en même temps qu'a sans doute augmenté la curiosité, voire la vigilance des membres de la Société des Amis des Sciences de Varsovie lors de la lecture de Podróż do Kalopei.

Sans s'y être nullement attendu, le voyageur polonais découvre en Australie un pays qui représente un État régulier, au sens social et politique du terme, mais qui, de plus, est habité et gouverné par... des Polonais. C'est d'ailleurs d'eux qu'il tire son nom : Kalopea vient du nom de son habitant, Kalop, c'est-à-dire Polak (Polonais) lu à l'envers. Son histoire remonte au temps et à la personne du roi de Pologne Boleslas II le Généreux (1040/1042-1081/1082), connu beaucoup mieux dans la tradition polonaise en tant que Boleslas le Téméraire, considéré coupable

${ }^{4} C f$. Muriel Proust de la Gironière, Nicolas Baudin. Marin et explorateur ou le mirage de l'Australie, Éditions du Gerfaut, Paris 2002 ; M. Jangoux, Le voyage aux Terres australes du commandant Nicolas Baudin. Genèse et préambule (1798-1800), PUPS, Paris 2013. 
de l'assassinat de l'évêque de Cracovie Stanislas de Szczepanów en $1079^{5}$. La légende veut que, suite à la mort de Stanislas, Boleslas, excommunié et menacé par la révolte des nobles et des prélats, ait dû abandonner la couronne et se soit enfui à la cour du roi Ladislas $\mathrm{I}^{\mathrm{er}}$ de Hongrie, ou, selon une autre version tout aussi incertaine mais beaucoup plus hagiographique, ait passé le reste de sa vie comme pénitent fervent pour racheter sa faute à l'abbaye bénédictine d'Ossiach en Carinthie (aujourd'hui en Autriche).

Dans son roman, Wojciech Gutkowski formule à ce propos une hypothèse historique tout à fait différente, aussi originale que complètement inattendue. Selon lui, forcé de fuir le pays, le roi Boleslas aurait quitté la Pologne ${ }^{6}$ avec un groupe de savants en vue de fonder un nouvel État quelque part dans le monde. Équipé de nombreux livres et d'outils, entouré d'« artisans, d'artistes et de fabricants » (PdK, p. 131) au nombre de plus de six cents personnes des deux sexes, le monarque polonais serait arrivé en Nouvelle Hollande en 1082, via l'Europe, la côte africaine et l'Océan Indien, pour fonder dans la partie centrale du continent australien un pays appelé la Kalopée. Six ans plus tard, le roi polonais, devenu entre-temps l'empereur Boleslas $\mathrm{I}^{\text {er }}$, aurait proposé en 1088 le texte de la constitution de l'État des Kalops, acceptée par tous avec beaucoup d'enthousiasme, et cette date aurait marqué l'an un de la nouvelle ère du calendrier des insulaires. On comprend qu'au moment de l'arrivée du voyageur polonais en Kalopée, ce dernier répétant d'ailleurs presque exactement l'itinéraire de Boleslas et de son groupe de migrants du $\mathrm{XI}^{\mathrm{e}}$ siècle, cet État fonctionne déjà depuis 714 ans en terre australe sans que personne en Europe ne puisse en deviner l'existence, ni surtout le repérer sur la carte géopolitique du monde.

Le narrateur nous le fait découvrir par le biais de deux moyens descriptifs différents, mais strictement complémentaires. D'abord, tout comme dans chaque narration autobiographique, le récit nous révèle autant les aventures que les observations du voyageur, mais il faut avouer que les premières sont quasi inexistantes dans cette partie du roman et cèdent visiblement la place à la description de tout ce que le narrateur observe et remarque dans le pays des Kalops. Il s'agit plutôt de décrire ce que l'on découvre dans l'espace kalopien que de raconter ses propres exploits, l'action du roman étant ainsi, conformément aux règles du récit utopique, entièrement soumise à l'observation et à la présentation qui en

${ }^{5}$ Sur la vie de Boleslas le Téméraire et l'histoire de son conflit avec l'évêque de Cracovie, voir surtout : S. Rosik, Bolesław Szczodry i jego czasy, Wydawnictwo Dolnośląskie, Wrocław 2002 ; T. Grudziński, Bolesław Śmiały-Szczodry i biskup Stanisław. Dzieje konfliktu, Universitas, Kraków 2010 ; N. Delestowicz, Bolesław II Szczodry. Tragiczne losy wielkiego wojownika (1040/1042-2/3 IV 1081 albo 1082), Avalon, Kraków 2016.

${ }^{6}$ Wojciech Gutkowski rejoint clairement ceux pour qui le crime commis par Boleslas semblerait justifié par la raison d'État, car dans son comportement (dont le romancier ne dévoile pourtant pas les détails), l'évêque Stanislas aurait offensé la dignité royale et aurait ainsi mérité son sort en tant que sujet de la monarchie ( $c f$. PdK, p. 131 ; voir aussi : P. Jasienica, Polska Piastów, PIW, Warszawa 1966, p. 104). 
découle directement ${ }^{7}$. Même les dialogues cités par le narrateur, aussi bien ses propres paroles que les propos de ceux qu'il rencontre sur son chemin, sont peu individualisés et prennent plutôt la forme d'exposés descriptifs qui servent plus à faire comprendre le sens du roman qu'à vivifier son action. Ensuite, à la fin de l'ouvrage, le narrateur présente in extenso la version intégrale de la constitution de l'État des Kalops élaborée jadis par l'empereur Boleslas Ir (PdK, pp. 172264). Composée de 169 articles répartis en 22 matières différentes (dont chacune porte un titre éclairant son contenu), la loi fondamentale des Kalops nous révèle en détail l'ensemble des principes qui fixent l'organisation et le fonctionnement de leur État. La lecture de la constitution nous permet de décrire ce dernier dans toutes ses dimensions et d'en saisir la forme socio-politique, mais aussi, de comprendre, comme le narrateur tient à nous l'annoncer dès la première page de son récit de voyage, qu'il s'agit en fait du " pays le plus heureux au monde, qui égale au paradis terrestre » (PdK, p. 55).

La source principale du bonheur de la Kalopée réside dans ce qui assure la dimension utopique du roman et, à la fois, constitue l'essence de l'idée de l'État que se forge Wojciech Gutkowski : il s'agit de l'inexistence absolue de la propriété individuelle. Celle-ci, considérée comme l'origine de tous les malheurs et de tous les vices possibles, tels les « vols, assassinats, tricheries, intrigues, injustices de toute sorte, flatteries, préjugés, superstitions, orgueil, guerres cruelles parmi les peuples » (PdK, p. 178, article 15 de la Constitution), est totalement remise en cause par l'auteur et bannie de son projet de société. Parti de la même conception du caractère pernicieux de la propriété que celle que l'on découvre chez un Rousseau, Gutkowski propose tout de même une solution politique beaucoup plus radicale à l'égard de l'idée de possession, qui n'est pas sans rappeler celle d'un Saint-Simon: «Toute propriété est une propriété publique qui appartient à tous et pour laquelle tous, sans aucune exception, doivent travailler » (PdK, p. 181, article 16 de la Constitution). L'auteur développe donc une vision utopique de pays communiste, au sens exact du terme, où, au nom du bonheur de tous les citoyens, la propriété privée est supprimée au profit de la propriété collective. L'idée de posséder n'existe pas, tout comme d'ailleurs l'argent, car les fruits du travail collectif - nourriture, chaussures et vêtements - sont distribués aux citoyens selon leurs besoins et mérites. Les Kalops vivent dans une communauté parfaite et peuvent jouir de deux autres droits fondamentaux dans leur pays, la liberté et l'égalité (PdK, p. 175, article 8 de la Constitution). Il s'agit d'une collectivité sans classes sociales, tous étant égaux et libres. On pourrait dire que, d'une certaine manière, le roman de Gutkowski renvoie au fameux paradoxe que révèle Rousseau au début de son Contrat social, lorsqu'il constate (livre I, chapitre I) que " l'homme est né libre et partout il est dans les fers ». Si Rousseau fait nettement la distinction

${ }^{7}$ Cf. A. Zgorzelski, Fantastyka, utopia, science-fiction. Ze studiów nad rozwojem gatunków, PWN, Warszawa 1980, p. 50. 
entre les lois justes et primordiales de la nature et celles, postérieures, de la société, qui ne font que détériorer les premières, l'auteur polonais propose une vision utopique d'un pays idéal où le social imite et préserve le naturel, les Kalops jouissant de la liberté et de l'égalité durant toute leur vie communautaire, car « ils naissent, vivent et meurent libres » (PdK, p. 175, article 8 de la Constitution). De toute évidence, Wojciech Gutkowski nous offre la vision d'un pays parfait, un vrai modèle de socialisme utopique à venir, où les habitants sont heureux, vivent en paix, travaillent pour le bien collectif ${ }^{8}$.

Tout en représentant une communauté utopique, la Kalopée possède pourtant, à l'instar de tout pays européen dit civilisé, tous les traits d'une organisation civile et politique régie selon des lois établies et des règles sociales concrètes. Comme il s'agit d'un État, elle a son propre système politique, un « gouvernement suprême ", autorité politique double car présidée par un empereur et un roi adjoint, et composé aussi de sénateurs, de députés et d'autres dignitaires publics (PdK, p. 192, article 50 de la Constitution). Le nombre de ces derniers doit refléter la structure administrative du pays, divisé en communes, districts et voïvodies. L'État a aussi sa capitale et ses armoiries : une représentation du Soleil qui doit obligatoirement être placée aux frontières du pays (ce qu'a aperçu le narrateur du roman lors de son arrivée en Kalopée) ainsi que sur différents établissements publics. Les origines polonaises du pays ne sont pas seulement rappelées dans son nom-anagramme officiel, mais aussi par la reconnaissance du polonais comme langue nationale que tous les Kalops doivent maitriser (PdK, p. 173, article 2 de la Constitution).

Même si la Kalopée est une entité politique sans classes sociales, il y existe onze différents degrés de dignité civile qui construisent une échelle sociale composée de fonctionnaires dont le rôle dans la communauté varie selon leur position et reste relatif aux degrés qu'ils possèdent. Contrairement à ce que l'on pourrait penser, l'idée de hiérarchie sociale qui résulte de celle des degrés ne va aucunement à l'encontre du principe d'égalité qui est l'un des piliers de l'État des Kalops. Ces derniers doivent passer très tôt, dès l'âge de 7 ans, par un système d'éducation nationale obligatoire organisé dans des académies publiques, destiné aux deux sexes, d'une durée de 15 ans pour les garçons et 10 ans pour les filles (PdK, p. 225, article 96 de la Constitution). Disposant ainsi des mêmes possibilités d'éducation, ils développent leurs talents naturels par un effort assidu, en forgeant leurs capacités intellectuelles et physiques qui, par nature, sont différentes. Ainsi s'agit-il d'avancer dans la société conformément à ce que la nature a fourni à l'homme en talents et aptitudes. Passer d'un degré de dignité civile à l'autre n'est donc rien d'autre que le résultat de l'essor de ses propres prédispositions

${ }^{8}$ Cf. W.M. Kozłowski, « Podróż do Kalopei. Pierwsza utopia socjalistyczna polska », Sfinks III, 1913, pp. 313-331 ; L. Guzicki, « Pierwsza polska utopia socjalistyczna », Międzyuczelniane zeszyty naukowe. Studia z historii myśli społeczno-ekonomicznej 7, 1964, Kraków, pp. 43-81. 
naturelles, et ne vient aucunement d'une injustice, terme qui tout simplement est inconnu en Kalopée. L'idée d'égalité des chances se laisse voir surtout dans le fait qu'aucun élève n'est dispensé de devoir travailler en même temps qu'il suit son éducation, ce qui fait que le voyageur polonais, quand il est entré en Kalopée, a rencontré sur son chemin des bergers qui savaient jouer de la flûte mieux que les musiciens européens. On lui a expliqué que chez les Kalops « celui qui aujourd'hui laboure et herse les champs, ou bien transporte du bois, demain donnera un concert, ou peindra une jolie toile » (PdK, p. 82).

Le pays utopique possède aussi une armée organisée selon une structure militaire bien précise qui garde une division nette en unités et grades, avec l'empereur comme chef suprême (PdK, pp. 244-245, articles 124 et 125 de la Constitution). Il ne s'agit pourtant pas de forces armées professionnelles. Comme tout citoyen masculin de la Kalopée doit se sentir soldat prêt à défendre sa patrie, l'État est capable, au cas où une agression viendrait de l'extérieur, d'organiser une armée puissante en décidant la levée en masse de quatre millions de soldats (PdK, p. 137). Il s'agit, bien entendu, d'un conflit où les Kalops devraient se défendre contre un ennemi malveillant, car eux-mêmes, en pacifistes ardents qu'ils demeurent, ne songeraient jamais à attaquer quelqu'un. Quant à leur religion, ils pratiquent le culte de l'Être Suprême. Ils ont des temples publics qui n'expriment aucune confession concrète, mais plutôt la religion naturelle, sans structure ni, surtout, sans prêtres. Il s'agit d'un modèle profondément déiste, où la croyance ne repose pas sur une religion révélée, mais sur une lumière naturelle, ce qui ne l'empêche guère de proposer un système de valeurs morales probantes universelles, que les Kalops suivent dans leur vie individuelle et civile (PdK, pp. 199-213, articles 66-74 de la Constitution). Jouissant d'un climat doux et possédant des terres fertiles, la Kalopée a des conditions naturelles idéales, propices au développement de l'agriculture, favorables aussi bien à la culture des blés qu'à l'élevage. La production agricole est extrêmement riche et apporte des quantités de nourriture suffisantes pour ravitailler toute la population, au point que le commerce n'existe pas chez les Kalops, ni à l'intérieur du pays, ni avec l'étranger, car le pays est autosuffisant (PdK, p. 255, article 145 de la Constitution). Le haut niveau de l'agriculture en Kalopée est sans doute dû au développement considérable des arts et des sciences, gage de progrès perpétuel du pays. Les travaux agricoles sont complètement mécanisés, grâce à quoi le taux de production est beaucoup plus élevé qu'en Europe. En outre, la médecine des Kalops connaît des remèdes contre des maladies qui sont toujours courantes en Europe, telles les « phtisie, tuberculose, hydropisie, gangrène, podagre, hémorroïdes » (PdK, pp. 87-88). Comme le remarque le voyageur polonais, « la physique, la chimie, la mécanique, l'hydraulique, la médecine et l'économie se trouvent à un tel point de développement qu'elles font toutes des miracles et il semble presque impossible d'imaginer que l'on puisse avancer encore » (PdK, p. 87). 
Certes, le roman de Gutkowski s'inscrit très fort dans la lignée de la littérature utopique qui, surtout depuis le fameux ouvrage de Thomas More (1478-1535) publié en latin à Louvain en 1516, a connu un essor considérable en Europe pendant les siècles suivants. Il reprend la plupart de ce que Jean-Michel Racault qualifie de constantes idéologiques et institutionnelles des utopies classiques, telles le communisme, l'égalitarisme, l'abolition de la propriété et de la monnaie ${ }^{9}$. On pourrait retrouver maintes ressemblances entre le roman de l'auteur polonais et ses prédécesseurs utopistes, à commencer par L'Utopie de More et son idée d'un État idéal situé sur une île. De plus, le motif du soleil, ainsi que l'idée d'égalité sociale, devraient inévitablement faire penser au fameux ouvrage utopique de Tommaso Campanella (1568-1639), Civitas Solis (La cité du Soleil), paru d'abord en latin en 1623 à Francfort. Ensuite, le roman de Gutkowski n'est pas sans rappeler celui de Denis Veirasse (1635-1700), Histoire des Sévarambes (Peuples qui habitent une partie du troisième continent, communément appelé la Terre Australe). Le fameux roman utopique de Veirasse, qui situe son récit en Australie, édité d'abord en anglais (I vol. en 1675, II vol. en 1679), maintes fois réédité et traduit en plusieurs langues (en français en 1682, en néerlandais en 1683, en allemand en 1689, en italien en 1728), connaît un grand succès vers la fin du XVII ${ }^{\mathrm{e}}$ siècle et tout au long de l'époque des Lumières. Certains chercheurs polonais vont aussi jusqu'à suggérer que Wojciech Gutkowski se serait probablement inspiré de deux ouvrages polonais de l'époque des Lumières : celui de Stanislas Leszczyński, Entretien d'un Européen avec un insulaire du royaume de Dumocala (1752), et le roman d'Ignacy Krasicki de 1776, Mikołaja Doświadczyńskiego Przypadki (Aventures de Nicolas Doświadczyński) ${ }^{10}$.

Une analyse plus détaillée de son ouvrage, à laquelle nous ne nous livrerons pas dans la présente étude, pourrait peut-être aider à démontrer si et dans quelle mesure Gutkowski se serait servi des textes mentionnés ci-dessus (ou d'autres ?) ${ }^{11}$, ce qui n'exclut guère une hypothèse contraire, selon laquelle les ressemblances de certains thèmes ou motifs ne seraient que purement fortuites ${ }^{12}$. Certes, la très bonne connaissance du français ${ }^{13}$, ainsi que l'accès à la très riche bibliothèque

${ }^{9}$ Cf. J.-M. Racault, op. cit., p. 383.

${ }^{10}$ Cf. J. Bartyś, op. cit., p. 167 ; Z. Gross, op. cit., p. 24.

11 Sans aucun doute, le roman de Gutkowski révèle aussi certaines ressemblances d'idées sociales et économiques avec la pensée de Gabriel Mably, d'Étienne-Gabriel Morelly ou de Jean Meslier. Voir à ce propos : Z. Najdowski, op. cit., pp. 135-168.

12 Par exemple, Julian Bartyś suggère que le motif du soleil présent dans le roman pourrait renvoyer moins à l'ouvrage de Campanella qu'au symbole solaire utilisé dans la loge maçonnique de Lublin, dont Gutkowski fut membre lors de son séjour dans cette ville (cf. J. Bartyś, op. cit., pp. 192-193).

${ }^{13}$ Wojciech Gutkowski a traduit en français l'ouvrage du sculpteur Jean-François Bosio, Traité élémentaire des règles du dessin (Paris 1800), ainsi que deux textes strictement militaires: Cours élémentaire de fortification (Paris 1806) du général Bellavène et De la défense des places fortes (Paris 1810) de Lazare Nicolas Carnot. 
(dont le fonds comptait plus de cinquante mille volumes) du comte Stanisław Kostka Zamoyski (1775-1856) lors du long séjour de Gutkowski à Zamość dans les années 1802-1812, permettent de supposer qu'il pouvait connaître les ouvrages de ses prédécesseurs utopistes. Néanmoins, au lieu de se lancer sur une voie de suppositions et d'hypothèses en vue d'établir des ressemblances ou des emprunts possibles, il semble beaucoup plus important de définir le véritable objectif de Podróż do Kalopei, c'est-à-dire de comprendre le rôle qu'assigne le romancier polonais à son propre texte. Il serait capital d'examiner la question de savoir si le récit utopique de Gutkowski possède les dimensions d'un ouvrage purement national, destiné seulement aux Polonais, et cela, au moment où leur pays n'existe plus sur la carte géopolitique de l'Europe, ou bien, au contraire, s'il tente de proposer à ses lecteurs potentiels une vision beaucoup plus universelle qui s'étend sur un espace beaucoup plus grand que celui de sa patrie.

Certes, le fait que le romancier ait rédigé son texte en polonais, sans s'efforcer en même temps de le traduire en français, devrait faire croire que c'est uniquement aux Polonais qu'il l'aurait adressé. De plus, le nom de la Kalopée sous lequel se cache, comme on le sait bien, celui du Polonais, ainsi que l'histoire romanesque de ce pays, qui remonte clairement à celle du monarque polonais Boleslas le Téméraire, constitueraient des arguments fort en faveur du caractère purement national du roman. Sans oublier que les habitants de cet État utopique situé dans la Terre Australe gardent le polonais comme langue maternelle, comme s'ils voulaient à tout prix préserver les liens avec leur pays d'origine. Certains chercheurs polonais vont même jusqu'à supposer que le texte de Gutkowski, écrit probablement avant 1817, aurait été conçu comme projet de constitution du Duché de Varsovie ou, après la chute de Napoléon, du Royaume de Pologne ${ }^{14}$.

Or, non seulement rien ne prouve de telles hypothèses, mais de plus, tout porte à croire que Gutkowski aurait plutôt voulu assurer à son texte un caractère universel, même si la Pologne lui sert de prétexte. Lorsque l'on se souvient de son appartenance à la franc-maçonnerie, on comprend beaucoup mieux que son projet de communauté idéale tient à surmonter les frontières des pays et des nationalités, car il s'applique à tous, indépendamment du lieu de naissance ${ }^{15}$. En maçon éclairé qu'il est, il veut avant tout décrire un État idéal, régi selon des lois qui sont hors du temps et hors du contexte géographique. Zygmunt Gross, grâce à qui nous avons reçu la première édition officielle du roman de Gutkowski, remarque avec justesse que les références à la Pologne — aussi bien à sa situation historique qu'à son état actuel - sont peu nombreuses, presque inexistantes, et cèdent visiblement

${ }^{14}$ Cf. W.M. Kozłowski, op. cit., pp. 318-319 ; J. Bartyś, op. cit., pp. 153-154.

15 Il serait d'ailleurs très intéressant d'analyser le roman de Gutkowski comme un ouvrage contenant un message occulte, de voir derrière différents symboles, noms propres, chiffres et nombres dont il se sert, un texte rédigé par un maçon à l'usage de ses frères. Une telle étude permettrait encore mieux de comprendre la dimension universalisante de Podróż do Kalopei. 
la place à des réflexions théoriques générales ${ }^{16}$. Ainsi donc, quoique le romancier polonais nous invite à un voyage en Kalopée, pays dont le nom rappelle l'origine de l'auteur presque autant que sa propre signature, la dimension universalisante de l'ouvrage n'en est pas pour autant moins évidente ${ }^{17}$.

Il s'agit d'ailleurs d'un trait caractéristique de tous les textes utopiques : même s'il est souvent possible de déchiffrer géographiquement l'espace décrit (par exemple l'Eldorado dans Candide de Voltaire, ou le pays des Troglodytes dans les Lettres persanes de Montesquieu), rien n'empêche de le percevoir comme un espace universel. Cela se laisse voir clairement dans le choix de l'Australie comme lieu de l'action du roman, cette île-continent ayant été un lieu privilégié du récit utopique tout au long du XVIII ${ }^{\mathrm{e}}$ siècle ${ }^{18}$. Le pays des Kalops forme un univers hermétique situé au milieu de l'espace inaccessible de la grande île du continent australien. Gutkowski propose une double forme insulaire, voire une véritable mise en abyme géographique, tout comme le fait Bernardin de Saint-Pierre dans son célèbre roman Paul et Virginie. L'Australie, encore mal connue, ou du moins, mal explorée à cette époque-là, se prête merveilleusement comme espace d'utopie qui permet à l'auteur de dessiner l'image d'une société idéale ${ }^{19}$, surtout grâce à son exotisme et par son éloignement de l'Europe ${ }^{20}$. Le pays des Kalops, contrairement à ce que pourrait suggérer l'origine polonaise de son nom, est celui de tous les êtres humains, tout comme chaque espace de la littérature utopique est censé être universel. Dans le cas du roman de Wojciech Gutkowski, c'est ce que semble nous dire clairement la colonne située à la frontière du pays, sur laquelle le nom de la Kalopée est exprimé en quatre langues, tout comme il existe quatre points cardinaux.

${ }^{16}$ Cf. Z. Gross, op. cit., pp. 32-33.

${ }^{17}$ En 1975, Janusz Marchewa a proposé l'hypothèse selon laquelle le roman de Gutkowski serait l'expression du messianisme romantique polonais, mais Zygmunt Najdowski a tout fait raison lorsqu'il constate que le chercheur polonais n'arrive guère à bien prouver sa conception millénariste (cf. J. Marchewa, « Podróż do Kalopei jak mesjanizująca utopia społeczna », Zeszyty Naukowe Uniwersytetu Łódzkiego, Seria I : Nauki Humanistyczno-Społeczne, z. 110, Filologia Polska, Łódź 1975, pp. 23-32 ; Z. Najdowski, op. cit., p. 16).

${ }^{18}$ Cf. J.-M. Racault, op. cit., pp. 381-383.

19 Pour Jean-Michel Racault, si « les utopies narratives classiques optent aussi massivement pour une localisation dans la fameuse "Terra Australis incognita", ou dans ses archipels satellites », c'est parce que «l'existence de ce troisième monde — après l'ancien et le nouveau — apparaît comme une quasi-certitude scientifique, tandis que l'inconnu géographique et humaine de ces territoires, que tout incite à supposer habités, légitime d'avance les fictions les plus hardies » (ibidem, pp. 382-383). Parmi les romanciers utopiques français qui, avant Gutkowski, ont contribué, avec Denis Veirasse, à renforcer le mythe des terres australes, il faut mentionner surtout : Gabriel de Foigny, La Terre Australe Connue (1676) et Simon Tyssot de Patot, Les voyages et aventures de Jacques Massé (1714).

${ }^{20}$ Cf. M. Bąk, Gdzie diabet (tasmański) mówi dobranoc. Wizerunek Australii w literaturze polskiej, Wydawnictwo Uniwersytetu Śląskiego, Katowice 2014, pp. 18 et 30-31. 


\title{
THE UTOPIAN JOURNEY OF WOJCIECH GUTKOWSKI: IS POLAND LOCATED IN AUSTRALIA?
}

\begin{abstract}
Summary
This article analyses the forgotten novel of Wojciech Gutkowski (1775-1826) Podróż do Kalopei [Trip to Kalopea], which was published in 1817. The story takes place in Australia, in a small country founded by the Polish king Bolesław II the Bold. Gutkowski describes the history of the Kalop nation (the name Kalop is an anagram of Polak), its social and political system where everyone is equal and the term private property does not exist. Despite the Polish background presented in the novel, the unique vision of the nation seems to express a universal message which is valid for all the countries. That is the reason why Gutkowski's work is considered to be one of the most important pieces of classical utopian literature.
\end{abstract}

Key words: Wojciech Gutkowski, utopian literature, Poland, Australia. 